\title{
Characterization of genes for the biosynthesis of the compatible solute ectoine from Marinococcus halophilus and osmoregulated expression in Escherichia coli
}

\author{
Petra Louis and Erwin A. Galinski \\ Author for correspondence: Erwin A. Galinski. Tel: +49228 733799. Fax: +49228737576.
}

Institut für Mikrobiologie \& Biotechnologie, Rheinische FriedrichWilhelms-Universität Bonn, Meckenheimer Allee 168, 53115 Bonn, Germany

\begin{abstract}
The genes of the biosynthetic pathway of ectoine (1,4,5,6-tetrahydro-2-methyl4-pyrimidinecarboxylic acid) from the Gram-positive moderate halophile Marinococcus halophilus were cloned by functional expression in Escherichia coli. These genes were not only expressed, but also osmoregulated in E. coli, as demonstrated by increasing cytoplasmic ectoine concentration in response to medium salinity. Sequencing of a $4.4 \mathrm{~kb}$ fragment revealed four major ORFs, which were designated ect $A$, ect $B$, ectC and orfA. The significance of three of these genes for ectoine synthesis was proved by sequence comparison with known proteins and by physiological experiments. Several deletion derivatives of the sequenced fragment were introduced into $E$. coli and the resulting clones were investigated for their ability to synthesize ectoine or one of the intermediates in its biosynthetic pathway. It was demonstrated that ectA codes for L-2,4-diaminobutyric acid acetyltransferase, ectB for L-2,4diaminobutyric acid transaminase and ectC for t-ectoine synthase. A DNA region upstream of ect $A$ was shown to be necessary for the regulated expression of ectoine synthesis in response to the osmolarity of the medium.
\end{abstract}

Keywords: Marinococcus halophilus, compatible solutes, ectoine genes, osmoregulation, salt stress

\section{INTRODUCTION}

Saline environments are characterized by high osmotic strength (low water potential). Most halophilic eubacteria cope with these conditions by accumulating small, highly water-soluble organic compounds, the so-called compatible solutes (Brown, 1976). These osmolytes enable organisms to adapt to a wide range of salt concentrations by adjusting the cytoplasmic solute pool to the osmolarity of the surrounding environment. Ectoines represent the predominant class of osmolytes in aerobic chemoheterotrophic eubacteria (Severin et al., 1992; Frings et al., 1993; Galinski, 1995). The biosynthetic pathway for ectoine has been elucidated at the enzymological level in Gram-negative eubacterial halophiles (Peters et al., 1990; Tao et al., 1992; Galinski \& Trüper, 1994). It comprises three steps, the first being the conversion of aspartate semialdehyde, an intermediate in amino acid metabolism, to L-2,4-diaminobutyric acid. This is followed by acetylation to $\mathrm{N}^{\gamma}$ -

The GenBank accession number for the sequence reported in this paper is U66614 acetyldiaminobutyric acid. The last step consists of a cyclic condensation reaction to form the tetrahydropyrimidine ectoine (Fig. 1).

Expression and regulation of genes involved in osmoadaptation has thus far been investigated almost exclusively in non-halophilic bacteria, especially Escherichia coli (for recent reviews see Csonka \& Hanson, 1991; Lucht \& Bremer, 1994; Galinski, 1995). This organism responds to increased salinity with the rapid accumulation of potassium and concomitant synthesis of glutamate as a counter anion. Subsequently, these charged solutes are partially replaced by endogenous trehalose or compatible solutes accumulated from the medium, if present (Dinnbier et al., 1988). Most studies at the molecular level have so far focused on the various solute uptake systems of E. coli (Altendorf \& Epstein, 1993; Mellies et al., 1995; Gowrishankar \& Manna, 1996). The only investigations concerned with the biosynthesis of compatible solutes covered choline oxidation and trehalose synthesis (Lamark et al., 1996; Strøm \& Kaasen, 1993). It has been proposed that relatively non-specific signals, such as intracellular 


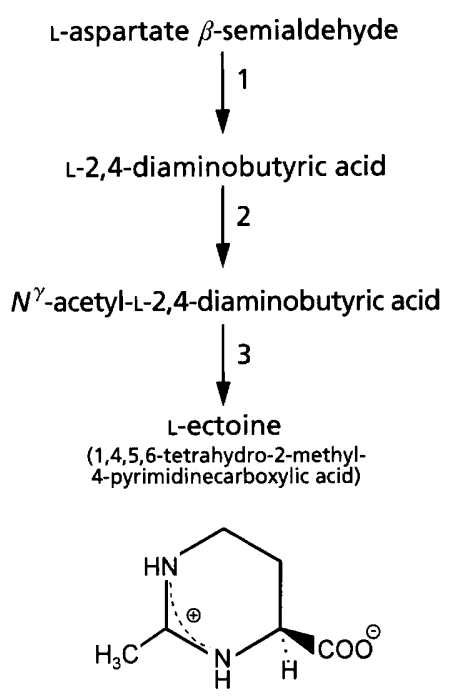

Fig. 1. Biosynthetic pathway for ectoine based on enzymological studies (Peters et al., 1990; Tao et al., 1992). 1, L2,4-diaminobutyric acid transaminase; 2, L-2,4-diaminobutyric acid $N^{\prime}$-acetyltransferase; 3 , L-ectoine synthase.

potassium glutamate concentration and DNA supercoiling (Booth \& Higgins, 1990), as well as specific regulatory mechanisms of the different genes investigated play a role in osmoregulation.

In this study, we report the identification and sequencing of the three genes responsible for the synthesis of the compatible solute ectoine from the Gram-positive halophile Marinococcus halophilus and their osmoregulated expression in E. coli. Our investigation provides, for the first time, an opportunity to compare the molecular organization of osmoregulatory elements from a true halophile with that of non-halophiles.

\section{METHODS}

Bacterial strains, growth conditions and plasmid. $M$. halophilus DSM $20408^{\mathrm{T}}$ was grown aerobically at $37^{\circ} \mathrm{C}$ in $\mathrm{LB}$ medium (Miller, 1972) containing $0.3 \%(\mathrm{w} / \mathrm{v})$ artificial sea salt and $3.7 \%(\mathrm{w} / \mathrm{v}) \mathrm{NaCl}$. E. coli XL1-Blue (Stratagene) was grown aerobically at $37^{\circ} \mathrm{C}$ either in LB medium or in medium MM63 (Larsen et al., 1987) with $1.5 \mathrm{ml}$ vitamin solution $\mathrm{l}^{-1}$ (Imhoff \& Trüper, 1977) and 1-5\% NaCl. To select for cells bearing plasmid pHSG575 and recombinant derivatives, the medium contained chloramphenicol at a final concentration of $25 \mu \mathrm{g} \mathrm{ml}^{-1}$. For the selection of cells containing recombinant plasmids on agar plates, isopropyl $\beta$-D-thiogalactopyranoside (IPTG, $0.5 \mathrm{mM}$ ) and 5-bromo-4-chloro-3-indolyl $\beta$-D-galactopyranoside (X-Gal, $40 \mu \mathrm{g} \mathrm{ml}^{-1}$ ) were added. For supplementation studies, MM63 with $2-3 \% \mathrm{NaCl}$ contained $2 \mathrm{mM}$ diaminobutyrate or $N^{\gamma}$-acetyldiaminobutyrate. At the end of the exponential growth phase, cells were harvested $(15000 \mathrm{~g}$, $\left.18^{\circ} \mathrm{C}\right)$ and freeze-dried.

Plasmid pHSG575 (Takeshita et al., 1987), a lacZ $\alpha$-complementing low-copy-number cloning vector, was kindly provided by E. Bremer (Universität Marburg, Germany).
DNA techniques. Genomic DNA of $M$. halophilus was prepared using Qiagen genomic-tips as described by the manufacturer. Small- and large-scale plasmid preparations of E. coli XL1-Blue were performed using Qiaprep spin columns and the Plasmid Midi kit from Qiagen, respectively.

For the construction of a library, genomic DNA from $M$. balophilus was partially digested with the restriction endonuclease Sau3A and separated on an agarose gel. Fragments ranging from 5 to $15 \mathrm{~kb}$ were recovered by electroelution and ligated into the dephosphorylated BamHI site of pHSG575 according to standard techniques (Ausubel et al., 1991). E. coli XL1-Blue was transformed with the recombinant plasmids by the $\mathrm{CaCl}_{2}$ method (Cohen et al., 1972).

Southern hybridization analysis was performed on nitrocellulose membranes (Schleicher \& Schuell) according to standard techniques (Ausubel et al., 1991) using the Nonradioactive Digoxigenin DNA Labeling and Detection kit from Boehringer Mannheim for the preparation of DNA probes.

Plasmids pOSM11, pOSM12, pOSM13, pOSM14 and pOSM18 were constructed by deletion of defined restriction fragments from pOSM1 and religation after the creation of blunt ends with the Klenow fragment of DNA polymerase I. Restriction enzymes and the Klenow fragment were purchased from Boehringer Mannheim with the exception of PpuMI, which was obtained from New England Biolabs.

Nested deletions for sequencing were prepared with the Double Stranded Nested Deletion kit (Pharmacia). Sequencing was performed with the AutoRead 1000 Sequencing kit and the automatic fluorescent sequencer ALF (Pharmacia) in one direction. Sequencing of the opposite strand was performed by Sequiserve (München, Germany).

DNA sequences were analysed with the programs GENEPRO, DNASIS and MACAW. Databank searches were carried out through the National Center for Biotechnology Information (NCBI) with the BLAST program (Altschul et al., 1990) and current versions of the available databases (October 1996).

Analytical methods. For the identification of intracellular solutes, freeze-dried cells were extracted with methanol/ chloroform/water $(10: 5: 4)$ by a modification of the technique of Bligh \& Dyer (1959), as described by Galinski \& Herzog (1990). Extracts were analysed by isocratic and gradient HPLC methods as described previously (Galinski \& Herzog, 1990; Kunte et al., 1993).

\section{RESULTS AND DISCUSSION}

\section{Cloning and osmotic expression of ectoine genes in E. coli}

E. coli is able to grow at salt concentrations of up to $3 \%$ $\mathrm{NaCl}$ in the minimal medium MM63 because of its intrinsic ability to adjust its cytoplasmic potassium glutamate and trehalose pools. At $5 \% \mathrm{NaCl}$, more efficient compatible solutes, such as betaine or ectoine, are needed for growth. Our aim was to provide $E$. coli XL1-Blue with genes for the synthesis of the compatible solute ectoine from M. halophilus, and thus increase its osmotolerance. To achieve this, colonies of E. coli XL1Blue carrying a genomic DNA library of $M$. halophilus were replica-plated onto medium MM63 containing 


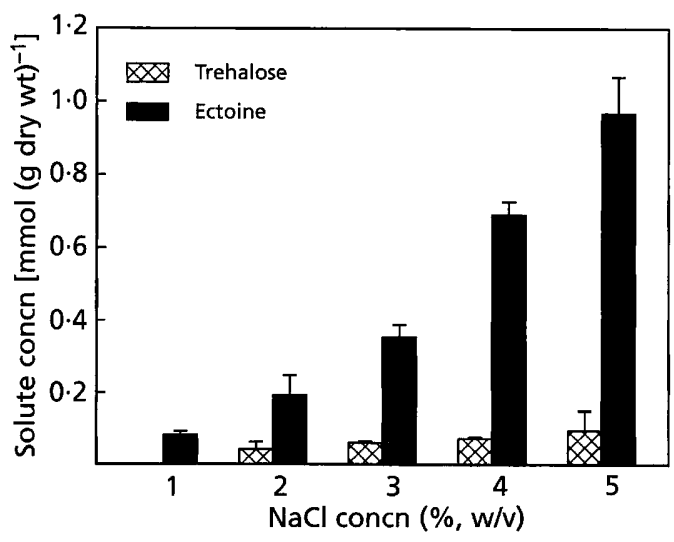

Fig. 2. Relationship between the intracellular solute concentration of $E$. coli(pOSM11) and medium salinity during growth in minimal medium MM63 as measured by isocratic HPLC. Mean values and standard deviations from three independent experiments are shown. Similar results were obtained with $E$. coli(pOSM1). Glutamate levels did not exceed $0.12 \mathrm{mmol}(\mathrm{g} \text { dry } \mathrm{wt})^{-1}$ regardless of the salt concentration of the medium. The trehalose pool of $E$. coli XL1-Blue in the presence of $3 \% \mathrm{NaCl}$ was $0.35 \mathrm{mmol}$ (g dry $\mathrm{wt})^{-1}$.

elevated salt concentrations and screened for clones with enhanced salt tolerance. One clone that was able to grow in the presence of $5 \% \mathrm{NaCl}$ was obtained. Plasmid preparations of this clone confirmed the presence of a recombinant plasmid, which we designated pOSM1.

To examine whether the enhanced salt tolerance of this clone was due to the synthesis of additional compatible solutes, the intracellular solute content was examined by HPLC. Besides trehalose, we detected the compatible solute ectoine in E. coli(pOSM1). Its cytoplasmic concentration increased with elevated salt concentrations to a maximum of $1 \mathrm{mmol}$ (g dry wt) $)^{-1}$ at $5 \% \mathrm{NaCl}$ (Fig. 2). At $3 \% \mathrm{NaCl}$ the cytoplasmic ectoine concentration was comparable to that gained through ectoine uptake in $E$. coli MC4100, as observed by Jebbar et al. (1992). Trehalose levels on the other hand were considerably lower in E. coli(pOSM1) [0.06 mmol (g dry wt $\left.)^{-1}\right]$ than in the ectoine-free host $E$. coli XL1-Blue $[0.35 \mathrm{mmol} / \mathrm{g}$ dry $w t)^{-1}$ ]. As we could not detect ectoine extracellularly, we conclude that ectoine was synthezised in an osmoregulated manner rather than simply excreted by the cells. In the complex medium LB containing $5 \%$ $\mathrm{NaCl}$, the observed biosynthesis of ectoine was suppressed almost completely (not shown). Instead, betaine was accumulated from the medium, which conforms with the current view that uptake of compatible solutes is preferred over biosynthesis (Dinnbier et al., 1988).

To confirm that the production of ectoine was dependent on plasmid pOSM1, we extracted it from the original isolate and retransformed it into E. coli XL1Blue, which then acquired osmotolerance by means of ectoine synthesis. Hybridization studies with the recombinant fragment of pOSM1 confirmed the presence of this particular sequence in genomic DNA of M. halophilus (not shown). This proves that the cloned DNA originated from M. halophilus.

\section{Sequencing of pOSM11}

Restriction analyses of pOSM1 revealed a recombinant fragment of $5.9 \mathrm{~kb}$. Following the deletion of a $1.5 \mathrm{~kb}$ EcoRI fragment, we were able to demonstrate that the resulting plasmid pOSM11, containing a $4.4 \mathrm{~kb}$ recombinant fragment, still displayed osmoregulated ectoine synthesis (Fig. 2).

The entire recombinant DNA fragment of pOSM11 was sequenced (Fig. 3). Four major ORFs, oriented in the same direction, were identified. They are predicted to encode proteins of 172, 427, 129 and 110 amino acids, with deduced molecular masses of 19385, 47192, 14796 and $13142 \mathrm{Da}$, respectively. All reading frames, which we designated ect $A$, ect $B$, ect $C$ and orf $A$, are preceded by putative ribosome binding sites (Fig. 3). Downstream of orfA lies the $5^{\prime}$-end of another ORF (orfB). The lacZ promoter of the vector is oriented in the opposite direction, and thus transcription of these reading frames from the lac $Z$ promoter can be excluded.

The gene for the ectoine synthase from Halomonas sp. had already been cloned by reverse translation and sequenced by Min-Yu et al. (1993). We compared their sequence with our ORFs and found $47 \%$ identity to ectC. An alignment is shown in Fig. 4. Thus, ectC seems to encode the ectoine synthase from M. halophilus.

Databank searches using the BLAST algorithm (Altschul et al., 1990) revealed a high similarity of ORF ectB to transaminases belonging to subgroup II (Mehta et al., 1993). All of these transaminases aminate a terminal oxo group. The same holds true for L-2,4-diaminobutyric acid transaminase in the biosynthetic pathway for ectoine. The highest similarity was observed with $\gamma$ aminobutyrate-, ornithine- and acetylornithine transaminases. An alignment is shown in Fig. 5. Of four residues found in all transaminases examined previously (Mehta et al., 1993), three were present in ectB (see $\boldsymbol{\nabla}$ in Fig. 5). The fourth, an arginine, which binds the $\alpha$ carboxylate of the substrate, is replaced by a lysine in $e c t B$. Studies with aspartate transaminase have shown that when arginine is replaced by lysine, which also carries a positive charge, enzyme activity is maintained, albeit at a strongly reduced rate (Inoue et al., 1989). Considering that replacement of arginine with lysine is likely to have a greater steric effect on $\alpha$-aminating enzymes like aspartate transaminase, this exchange may have less relevance for a $\gamma$-aminating transaminase, especially as this particular region is not very conserved (Fig. 5). From these sequence comparisons, we conclude that ect $B$ is the first gene in the biosynthetic pathway of ectoine, the transaminase converting aspartate- $\beta$-semialdehyde to diaminobutyric acid.

The ect $A$ gene revealed no sequence similarity to known proteins, whereas orf $A$ and the truncated $\operatorname{orf} B$ shared 
GGGTAGTACACGCAAGGATGGGACTCGTTTTGCAGGAATTTGTCCCGCCAATTGTTTCTGTATACAAAATGTTCGGCGGCGGCACATTCGGAGTGGAAT 100 ACAATGGTTTTCCTGGATTCATCTAAGGGATGCTGTCGAAATGATTCTTTTTGCAGCTACAAATAAAGAAATTAAAGGTGCATTGAACATTACTGCGCCG 200 CAACCGGCGCAGATGGAAACACTCGGACGTGAAATAGCGAAAGTGACTGGACGTCCGCACTGGCTTTCCGTGCCGTCATTTATTATTGATAAAATGCTCO 300 GGGAATGAGCGTAATGATCCTTGGTGGACAGAAGGTTATTCCGCAAAAAGCCTTGGATCATGGTTTTTCCTTCCACTTCCCAAGCCTCCAGCCTGGCCT 400

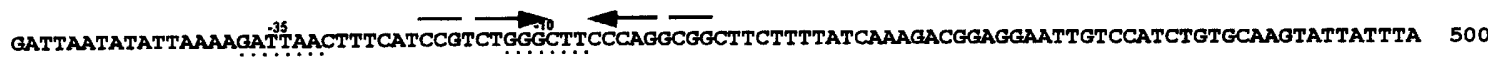
TCGTTTTCGATGTTCAGGTTACATAACACTCTATAAGTAGTAATAATACTCATAAATGACTGATAGGCAAGTTTGAAAATTTCCATAATTAACGTTAAGC 600

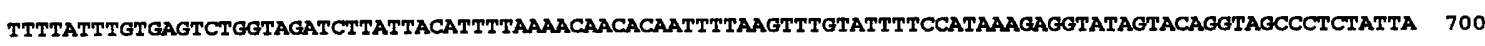
ecta

CTTTTAAA AGGTGGTAAGAAATGGAAACGAAAATGACTGGAACGAACGGTTCCGTCGATTCAATCGTTTMTGACAAACCAACCGTGGAAGACGGGGCAG BOO $\begin{array}{lllllllllllllllllllllllllllll}M & E & T & K & M & T & G & T & N & G & S & V & D & S & I & V & F & D & K & P & T & V & E & D & G & A & D\end{array}$

ACATGTGGGAACTTGTCAAAAACTCCACACTGGATTTAAATTCCTCATATAAGTATATTATGATGTGTGAATTTTTTGCCGAAACATGTGTTGTCOCTAA 900

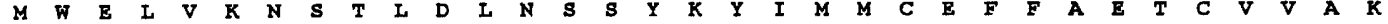

AGAAAACGATGAATTAGTGGGCTTCGTAACGGCCTTCATTCCACCTGAAAAACAGGATACCGTGTTTGTCTGGCAGGTGGGCGTAGATACTTCCCAACGG 1000

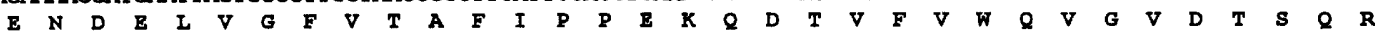

GGAAAAGTCTTGCTTCCCGTTTGCTGAACGCACTTTTAGAACGGATGTTTGTGAAAATGTGCTTTATTTGGAAGCAACTATCACCCCTTCTAATGAAG 1100

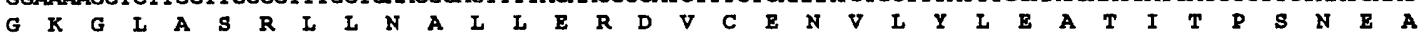

CATCCCAGGCTTTGTTTAAAAAGCTTGCCCAAAAAAGAGAAACTGAAGTGACGGTTTCTGAATGCTTTACGGAAGACCTTTTTCCAGATGACGAGCACGA 1200

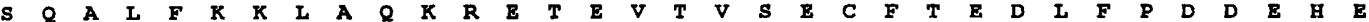
AGAAGAGTTG̈ E $E$ L T $T$ T $R$ I G P F ects

AACATCCCTA GGAGATAATTACTTTTATGATGCAGAATGATCTCAGCGTTTTTAATGAATACGAATCGGAAGTACGCAGTTATGTGACAGGATTCCCT 1400 $\begin{array}{llllllllllllllllllllllllllll}M & M & Q & N & D & L & S & V & F & N & E & Y & E & S & E & V & R & S & Y & V & R & G & F & P\end{array}$

ACCGTTTTCCACCAGGCAAAGGGGTATAAGCTTTGGGATCTGGACGGAAAGGAATATGTTGATTTCTTTTCCGGCGCCGGGGCCCTGAATTACGGTCATA 1500

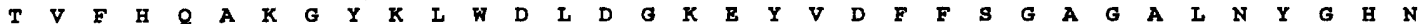

ATGATGAAAATATGAAAGAAAAGCTGCTTACCTATATTCAGGAAGACGGGTTACGCACTCCCTTGATATGGCAACTAAAGCTAAAGGCGAGTTCATCGA 1600

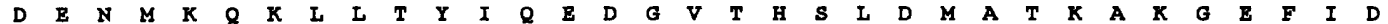

TGCTTTCCAAAATATTATTTTAAAGCCGCGTAATATGGATTATAAAATTATGTTCCCTGGCCCAACGGGCGCAAACAGCGTTGAAAGCGCCCTGAAGCTT 1700 $\begin{array}{lllllllllllllllllllllllllllllllllll}A & F & Q & N & I & I & I & K & P & R & N & M & D & Y & K & I & M & F & P & G & P & T & G & A & N & S & V & E & S & A & L & K & L\end{array}$

GCCCGGAAAGTAACCGGCCGGACAAACGTAGTCAGCTTTACTAATGGCTTCCACGGCATGACCATCGGTGCTTTGAGCGTTACCGGAAACAAATTCAAGC 180O $\begin{array}{lllllllllllllllllllllllllllllllllll}\mathbf{A} & \mathbf{R} & \mathbf{K} & \mathbf{V} & \mathbf{T} & \mathbf{G} & \mathbf{R} & \mathbf{T} & \mathbf{N} & \mathbf{V} & \mathbf{V} & \mathbf{S} & \mathbf{F} & \mathbf{T} & \mathbf{N} & \mathbf{G} & \mathbf{F} & \mathbf{H} & \mathbf{G} & \mathbf{M} & \mathbf{T} & \mathbf{I} & \mathbf{G} & \mathbf{A} & \mathbf{L} & \mathbf{S} & \mathbf{V} & \mathbf{T} & \mathbf{G} & \mathbf{N} & \mathbf{K} & \mathbf{F} & \mathbf{K} & \mathbf{R}\end{array}$

GTAACGGAGCCGGCATGCCTTTATCTAATACCTCTACGCTTCCGTATGACCAGTTCTTGAAGGAAAGCAATAATTCCATTGAATATATCGAGAACTTCCT 1900

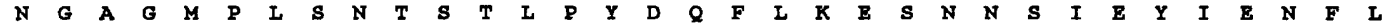

TGATAACGGAGGCAGCGGCCTCGATAAGCCGGCAGCGTTTATCGTAGAGACCGTACAGGGCGAAGGCGGCTTAAATGCTGCAAGCAGTGAATGGCTTCGT 200O

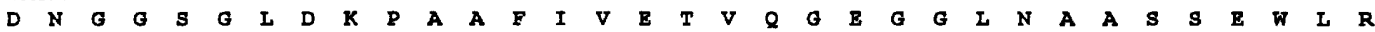

TCCATTGAAAAAATCTGCCGGGACGCGACATTAAGCTTATTCTGGACGATGTGCAGGCAGGCGTCGGCCGTACCGGTACTTTCTTCAGTTTTGAACCAG 2100

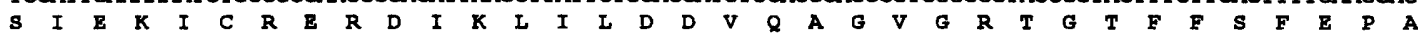

CTGGCATCAAACCTGACTTTGTATGTCTCTCCAAGTCCATCGGCGGCAATGGCTCACCGCTTGCCATTACTTTAGTGGCACCGGAATATGACAAATTTGC 2200 $\begin{array}{llllllllllllllllllllllllllllllllll}G & I & K & P & D & F & V & C & \text { L } & \text { S } & \text { K } & \text { S } & \text { I } & G & G & \text { N } & G & \text { S } & \text { P } & \text { L } & \text { A } & \text { I } & \text { T } & \text { L } & \text { V } & \text { A } & \text { P } & \text { E } & \text { Y } & \text { D } & \text { K } & \text { F } & \text { A } & \end{array}$

TCCTGGGGAGCACAACGGTACTTTCCGTGGAAATAATTTTGCTTTTGTAACAGGAACTGAAGCGTTGAATTACTGGAAAGACGACCGCCTGGAGAAAAAT 2300

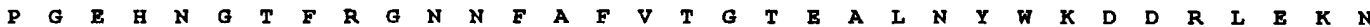

GTTCAGGAAAATCCGAGCGTATCACTTCTTTTCTCGATGACATGATTAAGAAGCACCCGGAAATGAAAGGTGTCCGTAAAGGGCGCGGCTTCATGCAGG 2400

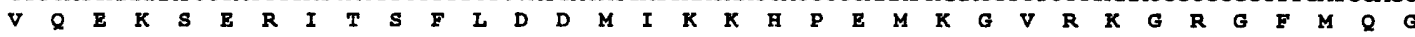

GTATCATGAGTCCTATCGAAGACCTGGCTGATAATATAGCGGGCAGATGCTTCGAGCACGGCCTAATTATGGAAACAGCTGGAGCGGAAGATGAAGTATT 2500

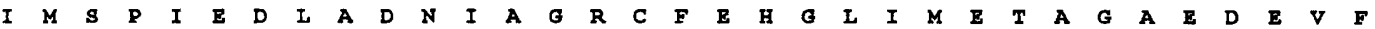

TAAGCTGTTCCCOCCAATCACAATCGATGATGAAGGGCTTGAGCGCGGCCTTTCAATTCTTCAGCAGGCAATTGAAGAAGTTACCGCTGAAAGCAACCTC 260O

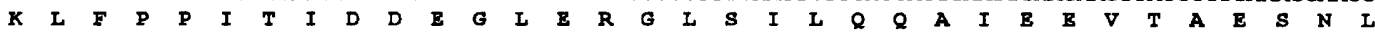

gTAGCCAAATAATCCACTGCATGACTAATTGATAAAAAGAAGCCGATCGATCGACTCCGGCGGTCGGCCTTTTTATGATATAAACTCTTAACTGACAAG 2700 $\checkmark$ A $K$ *

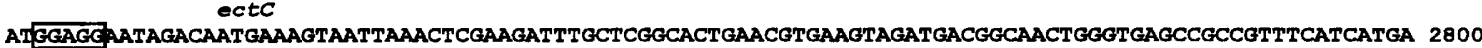

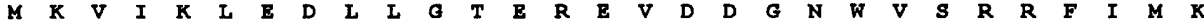

AAGACGACAACATGGGGTATTCGGTAAATGACACAATTATTCGTGCCGGTACTGAAACTCACATCTGGTACCAGAACCATCTCGAAACCGTATACTGCAT 2900

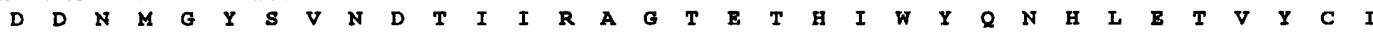

TGAAGGCGATGGAGAAATCGAAACTTTAAGCGCAATAAAGTATATCAGCTGGAACCAGGCGTATTATACGCACTCGATAAAAACGATGAACATATGCTT 3000

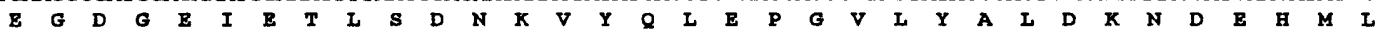

CGTGGAGGCAGCAAAGATATGCGTATGGTATGTGTCTTCAACCCGCCACTTAGCGGTCGTGAAGTGCATGACGAAAACGGTGTATATCCCGCTGATCTTG 3100 $\begin{array}{llllllllllllllllllllllllllllllllllll}R & G & G & S & K & D & M & R & M & V & C & V & F & N & P & P & I & S & G & R & E & V & H & D & E & N & G & V & Y & P & A & D & I & B\end{array}$

Fig. 3. For legend see facing page. 


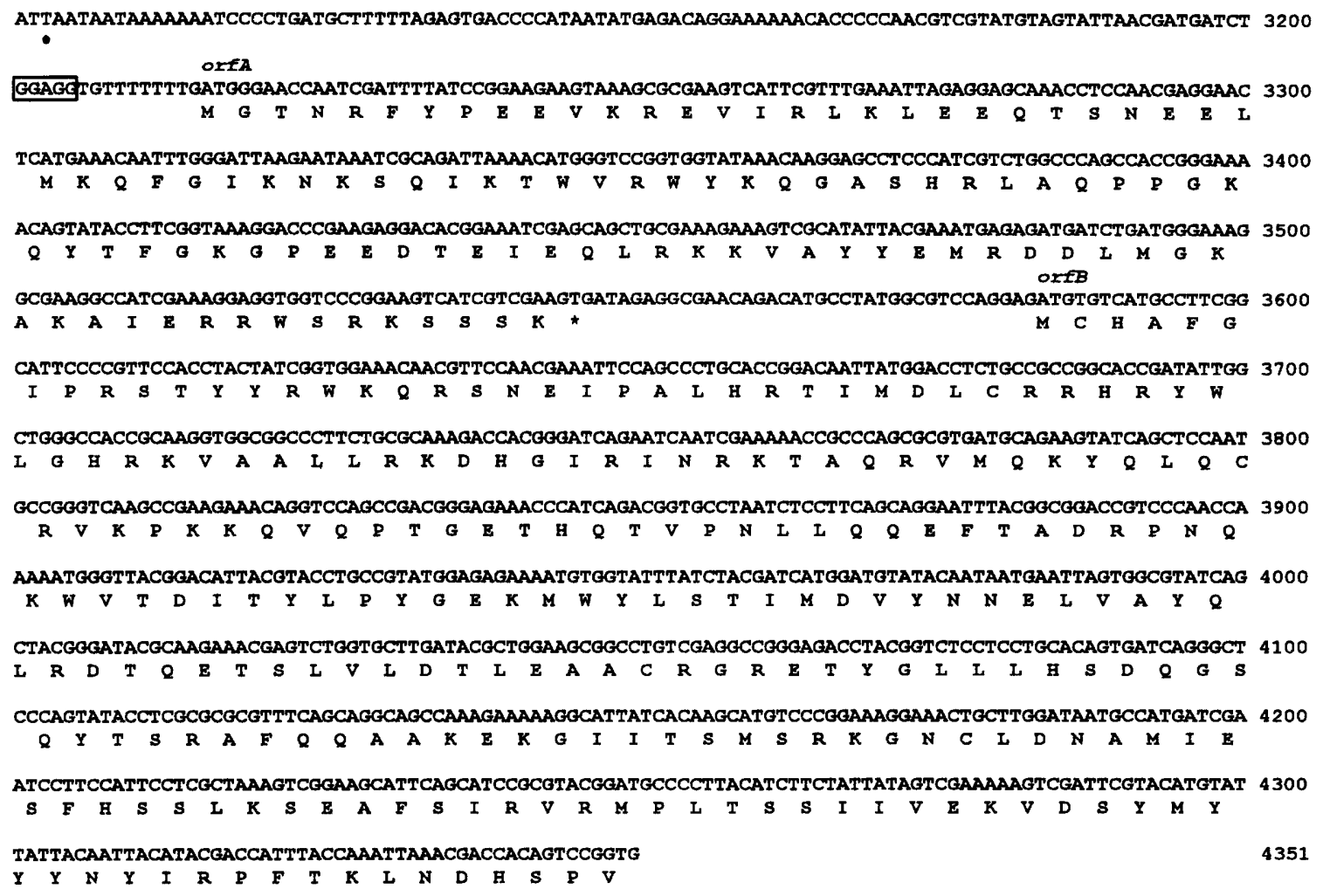

Fig. 3. Nucleotide sequence of the recombinant fragment of pOSM11. Deduced amino acid sequences of ORFs designated ect $A$, ectB, ectC, orf $A$ and orf $B$ ( $5^{\prime}$-end) are shown below the sequence. Stop codons are marked with asterisks. Putative ribosome-binding sites are boxed. Consensus sequences for two putative $\sigma^{70}$-dependent promoters (consensus TTGACA- $\mathrm{N}_{17}$-TATAAT) are underlined. Putative consensus sequences for a $\sigma^{\mathrm{B}}$-dependent promoter (consensus GTTIAA-N $_{12-14}$-GGGTAT) are marked with dotted lines. Palindromic sequences are marked by arrows.

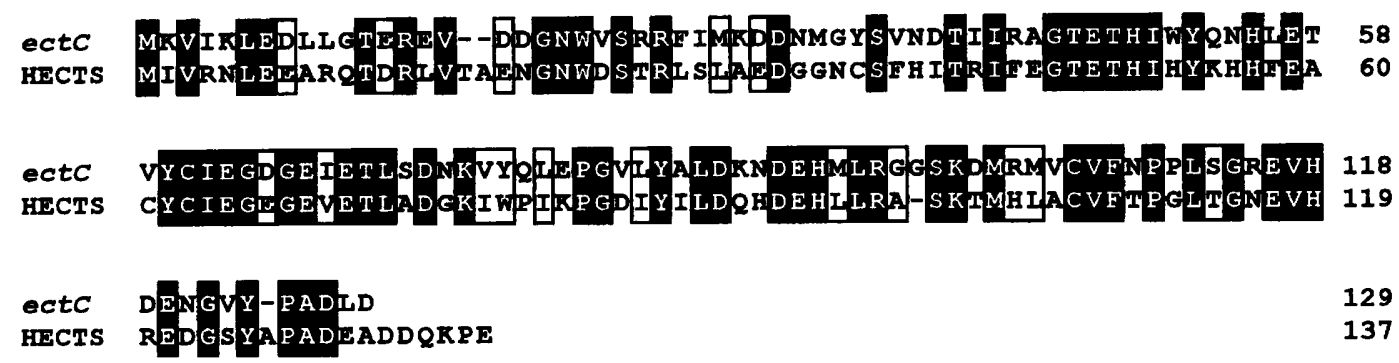

Fig. 4. Alignment of the sequences of reading frame ectC with the sequence of the ectoine synthase of a Halomonas sp. (HECTS) (Min-Yu et al., 1993). Identical positions are shown against a dark background and conservative replacements are boxed. The following were regarded as conservative replacements: R-K-H, D-E, N-Q, S-T, G-A, F-Y-W, I-L-V-M.

similarities with ORFs of the same name believed to encode transposases of IS elements.

\section{Ectoine synthesizing capacity of deletion derivatives of pOSM11}

In order to confirm the gene assignments based on sequence similarities and to determine the potential role of ect $A$ and orf $A$ in ectoine synthesis, we constructed several deletion derivatives of pOSM11 and examined them physiologically (Fig. 6). Analysis of pOSM12, which was deficient in the sequences 300 bp downstream of ectC and only retained a part of orf $A$, revealed the same osmoregulated pattern of ectoine synthesis as pOSM11 in E. coli XL1-Blue. This suggests that orfA is not involved in the ectoine biosynthetic pathway. pOSM13 carried a deletion within ect $C$ but still contained $e c t A$ and $e c t B$ and the sequences upstream of ect $A$. No ectoine synthesis was observed; instead the precursor $\mathrm{N}^{\gamma}$-acetyldiaminobutyric acid was accumu- 


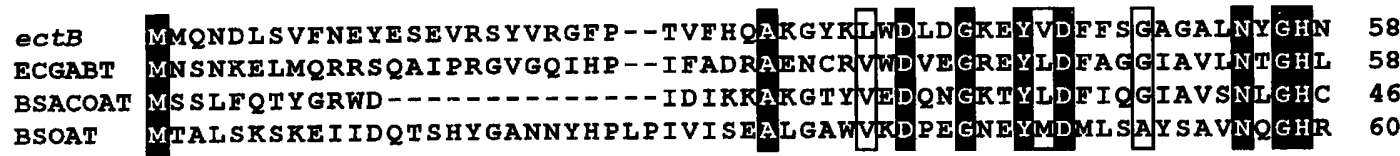

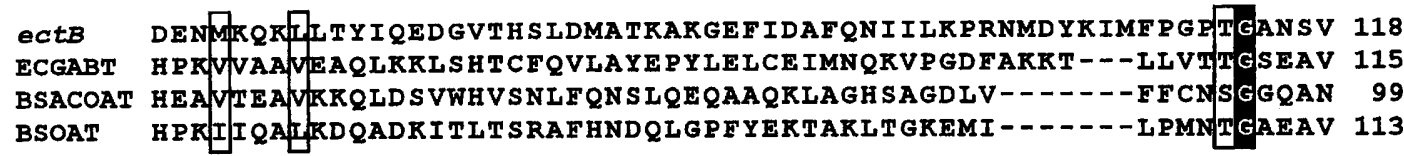

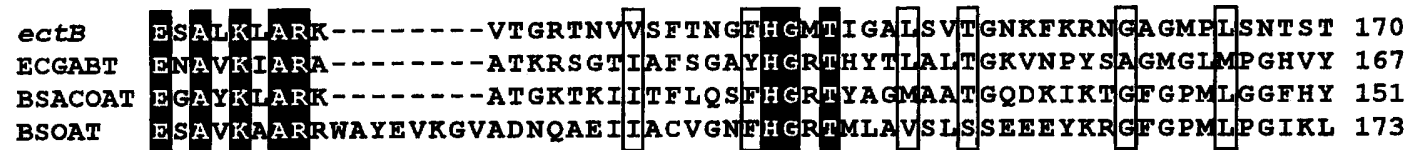

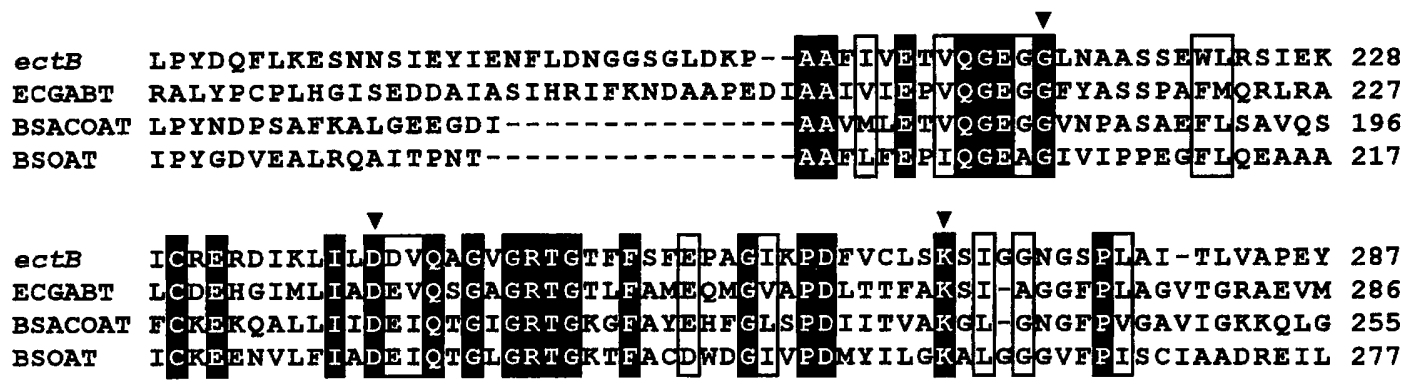

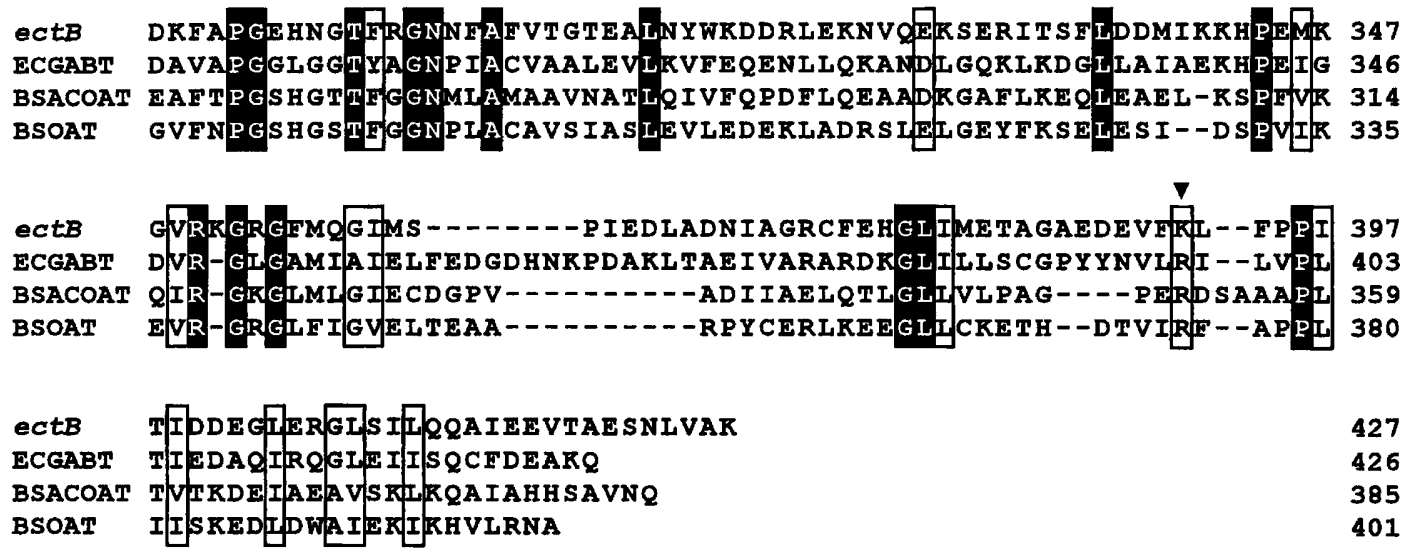

Fig. 5. Alignment of the sequences of reading frame ectB with transaminases. ECGABT, 4-aminobutyric acid transaminase from E. coli (Bartsch et al., 1990); BSACOAT, acetylornithine transaminase from B. subtilis (O'Reilly \& Devine, 1994); BSOAT, ornithine transaminase from B. subtilis (Gardan et al., 1995). Identical positions are shown against a dark background and conservative replacements are boxed. Conservative replacements are defined in the legend to Fig. 4 . $\nabla$, Residues identical in all transaminases examined so far according to Mehta et al. (1993).

lated. This confirms that $e c t C$ encodes ectoine synthase, which converts $N^{\prime}$-acetyldiaminobutyric acid to ectoine.

We constructed a subclone containing the complete ORF ectA and the upstream sequences (pOSM14). No accumulation of diaminobutyric acid or $N^{\gamma}$-acetyldiaminobutyric acid was observed, which is a further indication that $e c t B$ encodes the diaminobutyrate transaminase, the first enzyme in the ectoine pathway. When this subclone was supplemented with diaminobutyric acid, it synthesized $N^{\gamma}$-acetyldiaminobutyric acid, whereas E. coli XL1-Blue, containing only the vector pHGS575, was not able to acetylate diaminobutyric acid but simply accumulated it from the medium. This confirms our assumption that ectA is responsible for the acetylation step. In summary, our subcloning studies revealed that ect $A$ encodes diaminobutyric acid acetyltransferase, $e c t B$ diaminobutyric acid transaminase and ect $C$ ectoine synthase required for ectoine biosynthesis.

\section{Osmoregulation and genetic organization of ectoine} genes

Further deletion derivatives of pOSM11 were constructed to determine the approximate position of osmoregulating sequences in the promoter region. A 
(a)

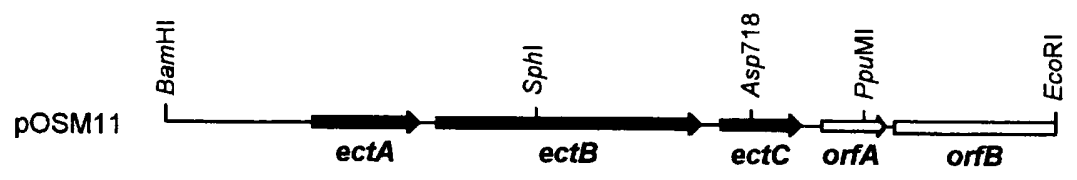

pOSM12

pOSM13

pOSM14

pOSM15

pOSM16

pOSM17

pOSM18

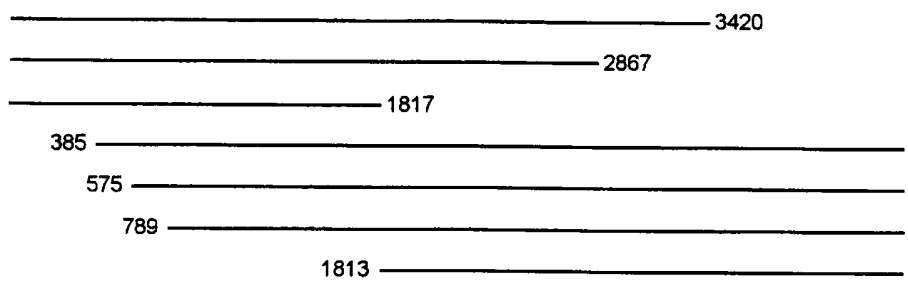

(b)

$\begin{array}{ccc}\begin{array}{c}\text { Supplementation } \\ \text { of culture }\end{array} & \text { biosynthesine } & \text { precursors } \\ - & \mathrm{R} & \text { inside cells }\end{array}$

$\begin{array}{ccc}- & R & - \\ - & - & \text { NACDABA } \\ \text { DABA } & - & \text { NACDABA } \\ - & R & - \\ - & U & - \\ - & - & - \\ \text { NACDABA } & - & \text { NACDABA }\end{array}$

Fig. 6. (a) Map of the sequenced DNA fragment of $M$. halophilus(pOSM11) and deletion derivatives. pOSM12, pOSM13, pOSM14 and POSM18 were constructed using the restriction sites indicated. pOSM15, pOSM16, and pOSM17 were generated with the Double Stranded Nested Deletion kit (Pharmacia). Numbers indicate the terminal positions (bp) of the subclones relative to pOSM11. Position of the ORFs in bp: ectA, 722-1240; ectB, 1329-2612; ectC, 2716-3105; orfA, 3216-3548; orfB, 3584-4351 (truncated). (b) Behaviour of the different subclones in physiological experiments. DABA, L2,4-diaminobutyric acid; NACDABA, $N^{\prime}$-acetyldiaminobutyric acid; R, osmoregulated; U, unregulated; -, none.

deletion of the first $384 \mathrm{bp}$ of the insert upstream of $e c t A$ (pOSM15, Fig. 6) had no effect on ectoine synthesis. Deleting a further $190 \mathrm{bp}$, however, apparently destroyed regulating sequences (pOSM16, Fig. 6), as the latter subclone showed constitutive ectoine synthesis. The cytoplasmic concentration ranged between 0.5 and $0.6 \mathrm{mmol}(\mathrm{g} \text { dry } \mathrm{wt})^{-1}$, independent of the salt concentration of the medium. It can therefore be concluded that osmoregulating sequences are located at least $150 \mathrm{bp}$ upstream of the first ORF.

Two possible consensus sequences for $\sigma^{70}$-dependent promoters were found upstream of ectB (Fig. 3). A search for consensus sequences of the osmoresponsive promoters for the compatible solute transport systems proU, proP and opuA (Mellies et al., 1994, 1995; Kempf \& Bremer, 1995) revealed no matches. As several osmoregulated genes in non-halophiles, including the genes for the biosynthesis of trehalose, are known to be under the control of $\sigma^{\mathrm{S}}$-dependent promoters (Strøm \& Kaasen, 1993; Gordia \& Gutierrez, 1996; Manna \& Gowrishankar, 1994; Mellies et al., 1995), we also conducted a search for consensus sequences of these (Strøm \& Kaasen, 1993), but were unable to find any matches. Instead, we found a sequence similar to the consensus for $\sigma^{\mathrm{B}}$ of Bacillus subtilis (Fig. 3) known to transcribe the so-called 'general stress proteins', which are induced by various stimuli, such as salt stress, ethanol treatment and starvation (Hecker et al., 1996). An imperfect palindrome lies within the probable -10 region.

A perfect palindrome downstream of ectA overlapping with one of the putative $\sigma^{70}$ promoter sequences (Fig. 3) may represent a Rho-dependent terminator. Therefore, the question arises as to whether the three genes are transcribed separately or as a single operon. The subclone E. coli(pOSM17), with a deletion of both the upstream sequences and part of ectA (Fig. 6), contained the whole reading frame ectB as well as the possible $\sigma^{70}$ consensus sequences and should therefore produce diaminobutyrate provided that $e c t B$ is transcribed from these putative promoters. However, we were not able to detect this compound inside the cells. Furthermore, another subclone containing pOSM18 (Fig. 6), which retained only the complete reading frame ect $C$, was also unable to produce ectoine when supplemented with $N^{\gamma}$ acetyldiaminobutyrate. These findings indicate that $e c t B$ and $e c t C$ are not transcribed independently. However, in order to ascertain that the ectoine genes are transcribed as a single operon, further studies at the RNA level are necessary.

In this study we have, for the first time, identified genes for the de novo biosynthesis of a compatible solute (ectoine) in addition to sequences necessary for its osmoregulated expression from a truly halophilic eubacterium. We were able to demonstrate that the osmoregulatory sequences of $M$. halophilus also function in E. coli. Earlier work on osmoregulated transport systems has also shown the potential of gene transfer. An osmoprotectant transporter from Erwinia chrysanthem $i$, encoded by the gene ous $A$ and analogous to proP from $E$. coli, displayed osmoregulated expression in $E$. coli, although no DNA homology upstream of the coding sequences of proP and ous $A$ was identified (Gouesbet et al., 1996). In addition, the betaine uptake system opuA from B. subtilis (Kempf \& Bremer, 1995) was also shown to be under osmotic control in E. coli. In this case, promoter consensus sequences resembling those of proP and proU were identified. These observations support the view that there are features in osmoregulation that are shared between different, even phylogenetically distant, bacteria. Further studies re- 
garding the osmoregulation of the ectoine genes from M. halophilus will provide a deeper insight into these mechanisms.

\section{ACKNOWLEDGEMENTS}

We would like to thank Erhard Bremer for providing plasmid pHSG575 and Martin Däumer for his valuable help with the sequencing. We also thank Christiane Dahl for her critical reading of the manuscript. This work was supported by the Deutsche Forschungsgemeinschaft $(\mathrm{Ga} 393 / 3)$ and by the European Union (Bio2-CT93-0274).

\section{REFERENCES}

Altendorf, K. \& Epstein, W. (1993). Kdp-ATPase of Escherichia coli. Cell Physiol Biochem 4, 160-168.

Altschul, S. F., Gish, W., Miller, W., Myers, E. W. \& Lipman, D. J. (1990). Basic local alignment search tool. J Mol Biol 215, 403-410.

Ausubel, F. M., Brent, R., Kingston, R. E., Moore, D. D., Seidman, J. G., Smith, J. A. \& Struhl, K. (1991). Current Protocols in Molecular Biology. New York: Wiley.

Bartsch, K., von Johnn-Marteville, A. \& Schulz, A. (1990). Molecular analysis of two genes of the Escherichia coli gab cluster: nucleotide sequence of the glutamate: succinic semialdehyde transaminase gene ( $g a b T)$ and characterization of the succinic semialdehyde dehydrogenase gene $(g a b D)$. J Bacteriol 172, 7035-7042.

Bligh, E. G. \& Dyer, W. J. (1959). A rapid method of lipid extraction and purification. Can J Biochem Physiol 37, 911-917.

Booth, I. R. \& Higgins, C. F. (1990). Enteric bacteria and osmotic stress: intracellular potassium glutamate as a secondary signal of osmotic stress? FEMS Microbiol Rev 75, 239-246.

Brown, A. D. (1976). Microbial water stress. Bact Rev 40, 803-846. Cohen, S. N., Chang, A. C. Y. \& Hsu, L. (1972). Non chromosomal antibiotic resistance in bacteria: genetic transformation of Escherichia coli by R-factor DNA. Proc Natl Acad Sci USA 69, 2110-2140.

Csonka, L. N. \& Hanson, A. D. (1991). Prokaryotic osmoregulation: genetics and physiology. Annu Rev Microbiol 45, 569-606.

Dinnbier, U., Limpinsel, E., Schmid, R. \& Bakker, E. P. (1988). Transient accumulation of potassium glutamate and its replacement by trehalose during adaptation of growing cells of Escherichia coli K-12 to elevated sodium chloride concentrations. Arch Microbiol 150, 348-357.

Frings, E., Kunte, H. J. \& Galinski, E. A. (1993). Compatible solutes in representatives of the genera Brevibacterium and Corynebacterium: occurrence of tetrahydropyrimidines and glutamine. FEMS Microbiol Lett 109, 25-32.

Galinski, E. A. (1995). Osmoadaptation in bacteria. Adv Microb Physiol 37, 273-328.

Galinski, E. A. \& Herzog, R. M. (1990). The role of trehalose as a substitute for nitrogen-containing compatible solutes (Ectothiorhodospira halochloris). Arch Microbiol 153, 607-613.

Galinski, E. A. \& Truper, H. G. (1994). Microbial behaviour in saltstressed ecosystems. FEMS Microbiol Rev 15, 95-108.

Gardan, R., Rapoport, G. \& Débarbouillé, M. (1995). Expression of the rocDEF operon involved in arginine catabolism in Bacillus subtilis. J Mol Biol 249, 843-856.

Gordia, S. \& Gutierrez, C. (1996). Growth-phase-dependent expression of the osmotically inducible gene osm $\mathrm{C}$ of Escherichia coli K-12. Mol Microbiol 19, 729-736.
Gouesbet, G., Trautwetter, A., Bonnassie, S., Wu, L. F. \& Blanco, C. (1996). Characterization of the Erwinia chrysanthemi osmoprotectant transporter gene ousA. J Bacteriol 178, 447-455.

Gowrishankar, J. \& Manna, D. (1996). How is osmotic regulation of transcription of the Escherichia coli proU operon achieved? Genetica 97, 363-378.

Hecker, M., Schumann, W. \& Völker, U. (1996). Heat-shock and general stress response in Bacillus subtilis. Mol Microbiol 19, $417-428$.

Imhoff, J. F. \& Truper, H. G. (1977). Ectothiorhodospira halochloris sp. nov., a new extremely halophilic phototrophic bacterium containing bacteriochlorophyll b. Arch Microbiol 114, 115-121.

Inoue, Y., Kuramitsu, S., Inoue, K., Kagamiyama, H., Hiromi, K., Tanase, S. \& Morino, Y. (1989). Substitution of a lysyl residue for arginine 386 of Escherichia coli aspartate aminotransferase. J Biol Chem 264, 9673-9681.

Jebbar, M., Talibart, R., Gloux, K., Bernard, T. \& Blanco, C. (1992). Osmoprotection of Escherichia coli by ectoine: uptake and accumulation characteristics. $J$ Bacteriol 174, 5027-5035.

Kempf, B. \& Bremer, E. (1995). OpuA, an osmotically regulated binding protein-dependent transport system for the osmoprotectant glycine betaine in Bacillus subtilis. J Biol Chem 270, 16701-16713.

Kunte, H. J., Galinski, E. A. \& Trüper, H. G. (1993). A modified FMOC-method for the detection of amino acid-type osmolytes and tetrahydropyrimidines (ectoines). J Microbiol Methods 17, 129-136.

Lamark, T., Røkenes, T. P., McDougall, J. \& Strøm, A. R. (1996). The complex bet promoters of Escherichia coli: regulation by oxygen (ArcA), choline (BetI), and osmotic stress. J Bacteriol 178, $1655-1662$.

Larsen, P. I., Sydne, L. K., Landfald, B. \& Strøm, A. R. (1987). Osmoregulation in Escherichia coli by accumulation of organic osmolytes : betaines, glutamic acid, and trehalose. Arch Microbiol $147,1-7$.

Lucht, J. M. \& Bremer, E. (1994). Adaptation of Escherichia coli to high osmolarity environments: osmoregulation of the highaffinity glycine betaine transport system ProU. FEMS Microbiol Rev 14, 3-20.

Manna, D. \& Gowrishankar, J. (1994). Evidence for involvement of proteins $\mathrm{HU}$ and RpoS in transcription of the osmoresponsive proU operon in Escherichia coli. J Bacteriol 176, 5378-5384.

Mehta, P. K., Hale, T. I. \& Christen, P. (1993). Aminotransferases: demonstration of homology and division into evolutionary subgroups. Eur J Biochem 214, 549-561.

Mellies, J., Brems, R. \& Villarejo, M. (1994). The Escherichia coli proU promoter element and its contribution to osmotically signaled transcription activation. J Bacteriol 176, 3638-3645.

Mellies, J., Wise, A. \& Villarejo, M. (1995). Two different Escherichia coli proP promoters respond to osmotic and growth phase signals. J Bacteriol 177, 144-151.

Miller, J. H. (1972). A Short Course in Bacterial Genetics: a Laboratory Manual and Handbook for Escherichia coli and Related Bacteria. Cold Spring Harbor, NY : Cold Spring Harbor Laboratory.

Min-Yu, L., Ono, H. \& Takano, M. (1993). Gene cloning of ectoine synthase from Halomonas sp. Annu Rep Int Center Cooperative Res Biotechnol, Jpn 16, 193-200.

O'Reilly, M. \& Devine, K. M. (1994). Sequence and analysis of the citrulline biosynthetic operon argC-F from Bacillus subtilis. Microbiology 140, 1023-1025. 
Peters, P., Galinski, E. A. \& Truper, H. G. (1990). The biosynthesis of ectoine. FEMS Microbiol Lett 71, 157-162.

Severin, J., Wohlfarth, A. \& Galinski, E. A. (1992). The predominant role of recently discovered tetrahydropyrimidines for the osmoadaptation of halophilic eubacteria. J Gen Microbiol 138, 1629-1638.

Strøm, A. R. \& Kaasen, I. (1993). Trehalose metabolism in Escherichia coli: stress protection and stress regulation of gene expression. Mol Microbiol 8, 205-210.

Takeshita, S., Sato, M., Toba, M., Masahashi, W. \& Hashimoto-
Gotoh, T. (1987). High-copy-number and low-copy-number plasmid vectors for $l a c Z$ alpha-complementation and chloramphenicol- or kanamycin-resistance selection. Gene 61, 63-74.

Tao, T., Yasuda, N., Ono, H., Shinmyo, A. \& Takano, M. (1992). Purification and characterization of 2,4-diaminobutyric acid transaminase from Halomonas sp. Annu Rep Int Center Cooperative Res Biotechnol, Jpn 15, 187-199.

Received 11 November 1996; accepted 21 November 1996. 